$\stackrel{W}{=}$

Global burnals Inc.

है

\title{
Fundamental Study of Quantitative Evaluation of Radio Wave Output of Wireless Medical Telemetry Transmitters Operable in Medical Field
}

\author{
By Kiyotaka Fujii, Kazumasa Kishimoto \& Munenori Inoue
} Abstract- Wireless medical telemetry systems (WMTSs) are important medical equipment for monitoring of biological information such as the electrocardiogram of a patient in a remote location in real time. However, because WMTSs have characteristically used channels that are unique to the radio wave spectrum, many institutions have not previously managed WMTSs. Therefore, we examined a method for quantitative evaluation of the radio wave output of a wireless medical telemetry transmitter (WMTT) that can even be implemented in the medical field. In the experiments, we demonstrated the possibility of use of the method for quantitative evaluation of WMTT radio wave output in the medical field using two types of radio wave propagation model: a free-space propagation model and a two-ray ground reflection model. To determine the reference threshold value for use of each model, a breakpoint was found to be important to grasp the change point between short distance and long distance. It was indicated that the measured value was lower than the theoretical value below the breakpoint, while the measured value was slightly higher than the theoretical value beyond the breakpoint.

Keywords: wireless medical telemetry transmitter, distance characteristics, free-space propagation model, two-ray ground reflection model.

GJMR-K Classification: NLMC Code: QS 26

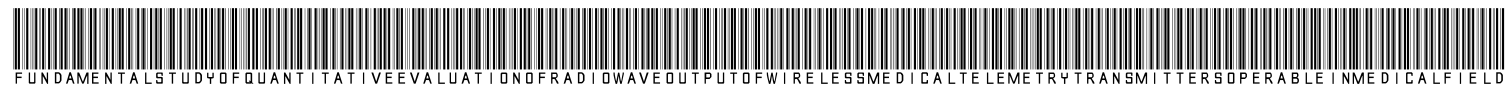

Strictly as per the compliance and regulations of:

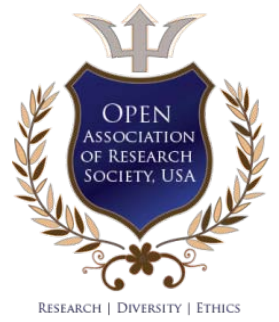

(c) 2020. Kiyotaka Fujii, Kazumasa Kishimoto \& Munenori Inoue. This is a research/review paper, distributed under the terms of the Creative Commons Attribution-Noncommercial 3.0 Unported License http://creativecommons.org/licenses/by-nc/3.0/), permitting all non-commercial use, distribution, and reproduction in any medium, provided the original work is properly cited. 


\title{
Fundamental Study of Quantitative Evaluation of Radio Wave Output of Wireless Medical Telemetry Transmitters Operable in Medical Field
}

\author{
Kiyotaka Fujii ${ }^{\alpha}$, Kazumasa Kishimoto ${ }^{\sigma}$ \& Munenori Inoue ${ }^{\rho}$
}

\begin{abstract}
Wireless medical telemetry systems (WMTSs) are important medical equipment for monitoring of biological information such as the electrocardiogram of a patient in a remote location in real time. However, because WMTSs have characteristically used channels that are unique to the radio wave spectrum, many institutions have not previously managed WMTSs. Therefore, we examined a method for quantitative evaluation of the radio wave output of a wireless medical telemetry transmitter (WMTT) that can even be implemented in the medical field. In the experiments, we demonstrated the possibility of use of the method for quantitative evaluation of WMTT radio wave output in the medical field using two types of radio wave propagation model: a free-space propagation model and a two-ray ground reflection model. To determine the reference threshold value for use of each model, a breakpoint was found to be important to grasp the change point between short distance and long distance. It was indicated that the measured value was lower than the theoretical value below the breakpoint, while the measured value was slightly higher than the theoretical value beyond the breakpoint.
\end{abstract}

Keywords: wireless medical telemetry transmitter, distance characteristics, free-space propagation model, two-ray ground reflection model.

\section{InTRODUCTION}

T he Ministry of Health, Labor and Welfare established medical equipment safety managers for all medical institutions in an April 2007 revision of medical law, along with training for the safe use of medical equipment, training for all medical equipment maintenance planning and implementation, and information gathering for safe use of the equipment [1]. Wireless medical telemetry systems (WMTSs) are important medical equipment that can monitor biological information such as the electrocardiogram of a patient in a remote location in real time. However, because WMTSs have characteristically used channels unique to the radio wave spectrum, many institutions have not previously managed WMTSs.

A report produced by the Electromagnetic Compatibility Conference Japan stated that although the introduction of WMTSs was particularly advanced in middle-and large-scale hospitals with more than 100

Author a $\sigma \rho:$ Kobe City Nishi-Kobe Medical Center, Kobe City, Japan. e-mails: sphu7xq9@gmail.com, ce.kazu@gmail.com,

qqcq5db9k@utopia.ocn.ne.jp beds, the overall implementation of the required radio channel management was only $48.1 \%$ and, even in the case of larger hospitals with 200 beds or more, only reached as high as $56.0 \%$ [2].

Examples of the main problems related to WMTS include radio waves not reaching their intended destinations, cross talk and electromagnetic noise, electromagnetic interference from other devices, and radio channel interference between proximal hospitals. These problems can be serious in some cases. A WMTS is generally configured as a complex system that includes a sensor, a wireless medical telemetry transmitter (WMTT), an antenna system, a patient monitor and other items of equipment. Therefore, in addition to the difficulties involved in maintenance management in the medical field, it has not been easy to investigate the cause when trouble occurs within these systems.

In previous research, although the electromagnetic interference between the WMTS and equipment such as a light-emitting diode (LED) has been demonstrated, the results presented did not represent a performance evaluation of the WMTT itself $[3,4,5]$. In addition, another report measured the reception limit distance to determine whether a signal can be received [6]. When using this reporting method, a space of approximately $100 \mathrm{~m}$ in the line of sight is required and such a space requirement is difficult to execute in the medical field. Additionally, the threshold of the transmission limit distance remains unclear and there is a high possibility that this threshold is influenced by artifacts such as obstacles.

Therefore, in this study, we aimed to clarify two points. The first point involved calculating the distance characteristics of the radio wave output from the WMTT using a simple radio wave propagation model and thus enabling derivation of the threshold value at each distance value. The second point involved was to evaluate the appropriateness of the threshold values derived as part of the first point by actually measuring the radio wave output of a WMTT used in the medical field. Based on the above, we then examined a method for quantitative evaluation of the radio wave output of a WMTT that can be implemented even in the medical field. 


\section{METHOD}

According to a standard produced by the Association of Radio Industries and Businesses, the value measured when a power meter is connected to an aerial terminal is defined as the aerial power ( $P$; units: $W$ ), and in the case of the class $A$ transmitter, which is mainly used in the medical field, $P$ is specified to be 1 $\mathrm{mW}$ or less [7].

Because it is difficult to measure the aerial power of the WMTT in the medical field, the voltage at the receiver input $(V ;$ units: $d B \mu V)$ at the spectrum analyzer was taken to be the radio wave output. In addition, because radio waves are attenuated over distance, the voltage received at the receiver input also varies with distance.

Therefore, to study the measurement conditions and the threshold value, the theoretical value was first calculated using the radio wave propagation model and the result was then compared with the actual measured value from radio wave output measurements in the medical field.

a) Distance characteristics of radio wave output when using the radio wave propagation model

When the unit conversion formulas (1) and (2) were used for conversion from power into voltage, $1 \mathrm{~mW}$ was converted into approximately $107 \mathrm{~dB} \mu \mathrm{V}$ for a system with $50 \Omega$ impedance ( $R$ unit: $\Omega$ ).

$$
\begin{gathered}
\mathrm{dB} \mu \mathrm{V}=20 \log _{10}\left(\frac{\mathrm{V}}{1 \mu \mathrm{V}}\right) \\
\mathrm{dB} \mu \mathrm{V}=20 \log _{10}\left(\sqrt{\mathrm{RP}} \times 10^{6}\right)
\end{gathered}
$$

For the radio wave propagation model, both the free-space propagation model and the two-ray ground reflection model were used. By subtracting the propagation loss derived from each model from the 107 $\mathrm{dB} \mu \mathrm{V}$ value calculated above, the distance characteristics of the radio wave output were acquired. The propagation distances were set at $0.1 \mathrm{~m}, 0.3 \mathrm{~m}, 0.5$ $\mathrm{m}, 1.0 \mathrm{~m}$, and $3.0 \mathrm{~m}$, and over the range from $5.0 \mathrm{~m}$ to $50 \mathrm{~m}$ in steps of $5.0 \mathrm{~m}$.

\section{i. Free-space propagation model}

The transmission power $\left(P_{t}\right)$ and the reception power $\left(P_{r}\right)$ are calculated from the transmission formula of the Friis Transmission Equation using the antenna gains $\left(G_{t}, G_{r}\right)$, the propagation distance $(d)$, and the wavelength $(\lambda)$ [8]. The free space radio propagation loss $\left(L_{f}\right)$ was calculated from equation (4). Assuming that isotropic antennas were used for both the transmitting and receiving antennas, the basic freespace model propagation loss $\left(L_{B} ;\right.$ units: $\left.d B\right)$ was calculated using equation (5).

$$
\frac{\mathrm{P}_{\mathrm{r}}}{\mathrm{P}_{\mathrm{t}}}=\frac{1}{\mathrm{~L}} \mathrm{G}_{\mathrm{r}} \mathrm{G}_{\mathrm{t}}
$$

$$
\begin{gathered}
\mathrm{L}_{\mathrm{f}}=\left(\frac{4 \pi \mathrm{d}}{\lambda}\right)^{2} \\
\mathrm{~L}_{\mathrm{B}}=10 \log _{10}\left(\frac{4 \pi \mathrm{d}}{\lambda}\right)^{2}
\end{gathered}
$$

\section{ii. Two-ray ground reflection model}

In line-of-sight propagation, the two-ray ground reflection model can be expressed using equations (6) and (7) using the transmission power and the reception power (i.e., $P_{t}$ and $P_{r}$, respectively). $\Delta \phi$ is the phase difference due to the distance difference between the two radio waves, and $h_{t}$ and $h_{r}$ are the transmitting and receiving antenna heights, respectively [9]. The break point $\left(d_{b}\right)$ at which the distance attenuation tendency changes is defined by equation (8). The distance attenuation was calculated using equation (9) for short distances and equation (10) for long distances with the break point acting as the boundary. For short distances from the break point, the direct wave and the reflected wave are both fading, but in this case, the average value was used.

Assuming that isotropic antennas were used for both the transmitting and receiving antennas, the basic radio wave propagation losses $\left(L_{t 1}, L_{t 2}\right.$; units: $\left.d B\right)$ of the two-ray ground reflection model were then calculated.

$$
\begin{gathered}
\frac{\mathrm{P}_{\mathrm{r}}}{\mathrm{P}_{\mathrm{t}}}=\left\{\frac{\lambda}{2 \pi \mathrm{d}} \sin \left(\frac{\Delta \varphi}{2}\right)\right\}^{2} \mathrm{G}_{\mathrm{r}} \mathrm{G}_{\mathrm{t}} \\
\Delta \varphi \approx \frac{4 \pi \mathrm{h}_{\mathrm{r}} \mathrm{h}_{\mathrm{t}}}{\lambda \mathrm{d}} \\
\mathrm{d}_{\mathrm{b}}=\frac{4 \mathrm{~h}_{\mathrm{r}} \mathrm{h}_{\mathrm{t}}}{\lambda} \\
\mathrm{L}_{\mathrm{t} 1}=10 \log _{10} 2\left(\frac{2 \pi \mathrm{d}}{\lambda}\right)^{2} \\
\mathrm{~L}_{\mathrm{t} 2}=10 \log _{10}\left(\frac{\mathrm{d}^{2}}{\mathrm{~h}_{\mathrm{r}} \mathrm{h}_{\mathrm{t}}}\right)^{2}
\end{gathered}
$$

For both models, the WMTT transmission frequency was calculated to be $448.8000 \mathrm{MHz}$.

b) Radio wave output measurement experiment in the medical field

To simulate line-of-sight propagation in the medical field, a measurement experiment was conducted in a corridor on the $3^{\text {rd }}$ floor of our hospital. External waves that could interfere with the operating frequency range $(420 \mathrm{MHz}$ to $450 \mathrm{MHz}$ ) of the WMTT from the exterior have not been confirmed. We measured the radio wave output of the WMTT (ZS-630P, Nihon Kohden, Co., Ltd.) using a spectrum analyzer (HAS9101B, Willtek). The specifications of this WMTT 
are listed in Table 1. The transmission frequency of the WMTT was $448.8000 \mathrm{MHz}$.

The spectrum analyzer and the WMTT were installed at a height above the floor of $1 \mathrm{~m}$ (Fig.1), and the distance characteristic of the WMTT was set to be equal to the distance characteristic of the radio wave output obtained using the radio wave propagation model (Fig.2: Point A to point B). Measurements were performed with Max Hold after sweeping for $30 \mathrm{~s}$ and were acquired three times for each propagation distance. The measurement data were first converted into an antilogarithm and then the data from the three measurement sets were averaged and expressed in $\mathrm{dB} \mu \mathrm{V}$.

Table 1: Specifications of the wireless medical telemetry transmitter

\begin{tabular}{|l|l|}
\hline \multicolumn{1}{|c|}{ Specification } & \multicolumn{1}{c|}{ Value } \\
\hline Operation mode & Type-A (digital) \\
\hline Modulation method & Frequency shift keying (FSK) \\
\hline Type of radio wave & F7D \\
\hline Center frequencies & $\begin{array}{l}420 \text { to } 440 \mathrm{MHz} \\
\text { (Channel changeable type) }\end{array}$ \\
\hline Occupied bandwidth & $8.5 \mathrm{kHz}$ \\
\hline Transmitting antenna & $\begin{array}{l}\text { Electrode lead, Built in, } \\
\text { Probe for oxygen saturation } \\
\text { rate in blood }\end{array}$ \\
\hline Output power & $1 \mathrm{~mW}$ \\
\hline
\end{tabular}

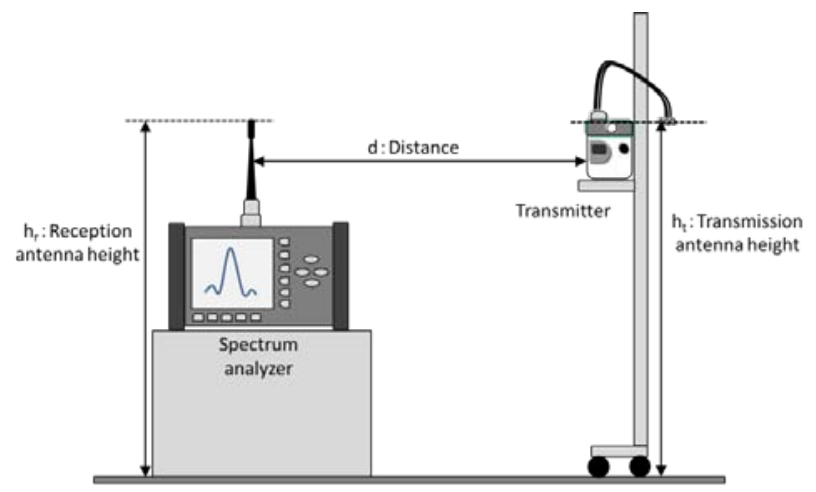

Fig.1: Schematic of radio wave output measurement experiment.

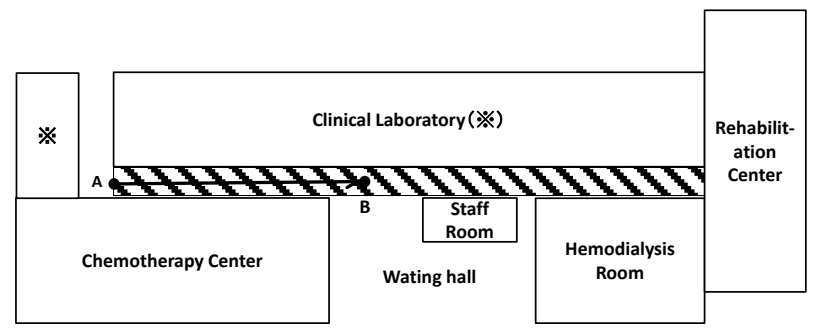

Fig. 2: Radio wave output measurement experiment in the medical field.

\section{Results}

a) Distance characteristics of radio wave output using the radio wave propagation model

We show the distance characteristics of the radio wave output that were determined using the radio wave propagation model in Fig.3, and the corresponding values are presented in Table 2. Because the break point is approximately $6.0 \mathrm{~m}$, the formula for $L_{t 1}$ was used for the distances up to $5.0 \mathrm{~m}$ and the formula for $L_{t 2}$ was used for the distances of $10 \mathrm{~m}$ and over. When $L_{B}$ and $L_{t 1}$ were compared, their tendencies were the same, with the intercept component of LB being only approximately $3 \mathrm{~dB}$ larger than that of Lt1 (equations (5) and (9), respectively). In contrast, when $L_{B}$ and $L_{t 2}$ were compared, $L_{t 2}$ increased abruptly with $d^{4}$ with respect to the increase with $d^{2}$ for LB (equations (5) and (10), respectively). Therefore, the radio wave output was reversed after the break point. 


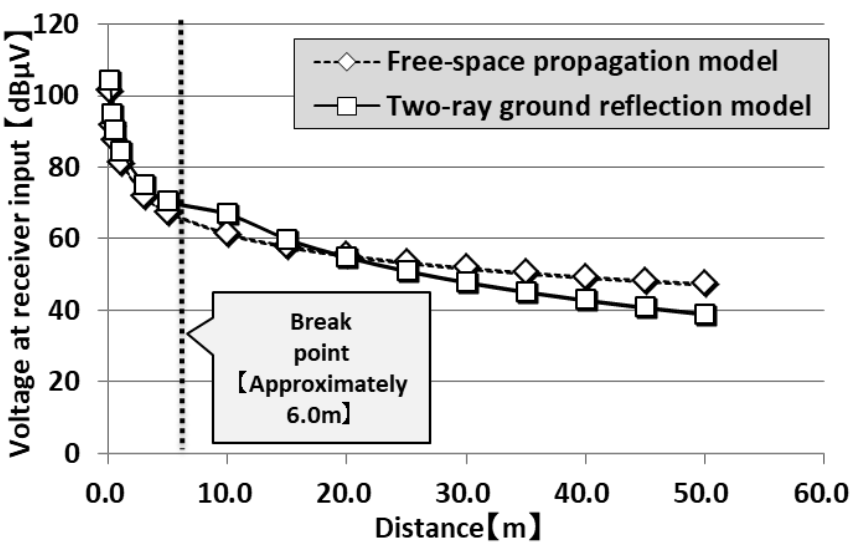

Fig. 3: Comparison of distance characteristics of the radio wave output when using the radio wave propagation model.

b) Radio wave output measurement experiment in the medical field

We show the relationship between the distance characteristics of the actual value of the radio wave output in the medical field and the radio wave output determined using the radio wave propagation model here (Fig.4).The corresponding numerical values are presented in Table 2.In both the free-space propagation model and the two-ray ground reflection model, reversal of the measured value in the medical field occurred after the breakpoint.
We also present the results of a regression analysis of the measured values in the medical field and those from the radio wave propagation model (Fig.5).

In addition, when we investigated the correlation between the free-space propagation model and the measured values in the medical field, a regression equation of $y=0.6177 x+24.629 \quad\left(R^{2}=0.911\right) \quad$ was obtained.

Similarly, for the relationship between the tworay ground reflection model and the measured value in the medical field, a regression equation of $y=0.5185 x+31.436\left(R^{2}=0.9354\right)$ was obtained.

Table 2: Radio wave outputs obtained using the radio wave propagation model and measured values in the medical field

\begin{tabular}{|c|c|c|c|c|c|c|}
\hline \multirow{2}{*}{$\begin{array}{l}\text { Distance } \\
\text { 【m】 }\end{array}$} & \multirow{2}{*}{$\begin{array}{c}\text { Free-space } \\
\text { propagation } \\
\text { model[dB } \mu \mathrm{V}]\end{array}$} & \multirow{2}{*}{$\begin{array}{c}\text { Two-ray ground } \\
\text { reflection model } \\
\lfloor\mathrm{dB} \mu \mathrm{V}\rfloor\end{array}$} & \multicolumn{4}{|c|}{$\begin{array}{c}\text { Measured value in medical field } \\
\lfloor\mathrm{dB} \mu \mathrm{V} \backslash\end{array}$} \\
\hline & & & $1 \mathrm{st}$ & 2nd & $3 \mathrm{rd}$ & Avereage \\
\hline 0.1 & 101.5 & 104.5 & 90.2 & 88.9 & 88.5 & 89.2 \\
\hline 0.3 & 92.0 & 95.0 & 81.6 & 83.6 & 80.2 & 81.9 \\
\hline 0.5 & 87.5 & 90.6 & 74.4 & 76.6 & 78.8 & 76.8 \\
\hline 1.0 & 81.5 & 84.5 & 72.8 & 68.3 & 69.9 & 70.5 \\
\hline 3.0 & 72.0 & 75.0 & 65.7 & 69.3 & 65.5 & 67.0 \\
\hline 5.0 & 67.5 & 70.6 & 67.5 & 67.0 & 66.3 & 66.9 \\
\hline 10.0 & 61.5 & $\begin{array}{l}67.0 \\
\end{array}$ & 64.5 & 64.4 & 64.5 & 64.5 \\
\hline 15.0 & 58.0 & 60.0 & 66.6 & 66.3 & 66.4 & 66.4 \\
\hline 20.0 & 55.5 & 55.0 & 62.3 & 63.8 & 64.5 & 63.6 \\
\hline 25.0 & 53.6 & 51.1 & 62.2 & 62.8 & 62.6 & 62.5 \\
\hline 30.0 & 52.0 & 47.9 & 57.3 & 56.7 & 57.4 & 57.1 \\
\hline 35.0 & 50.6 & 45.2 & 54.0 & 54.8 & 53.8 & 54.2 \\
\hline 40.0 & 49.5 & 42.9 & 54.4 & 53.5 & 53.1 & 53.7 \\
\hline 45.0 & 48.5 & 40.9 & 49.2 & 49.4 & 49.6 & 49.4 \\
\hline 50.0 & 47.5 & 39.0 & 50.7 & 48.7 & 50.8 & 50.1 \\
\hline
\end{tabular}



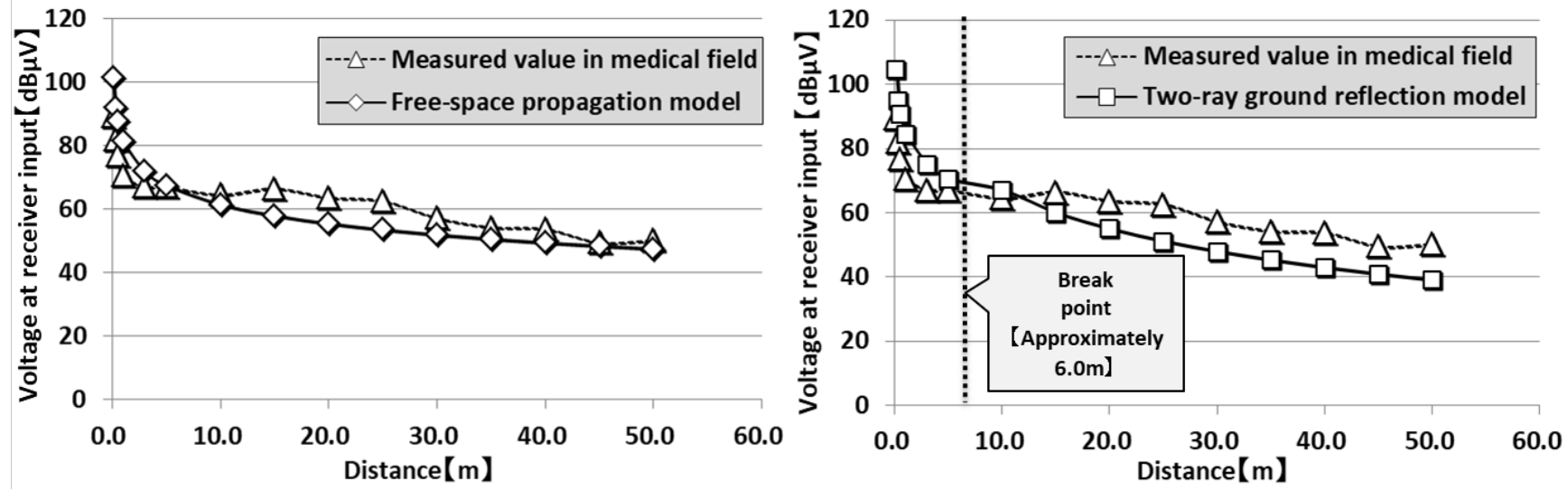

Fig. 4: Comparison of distance characteristics of the measured values in the medical field and those from the radio wave propagation model.
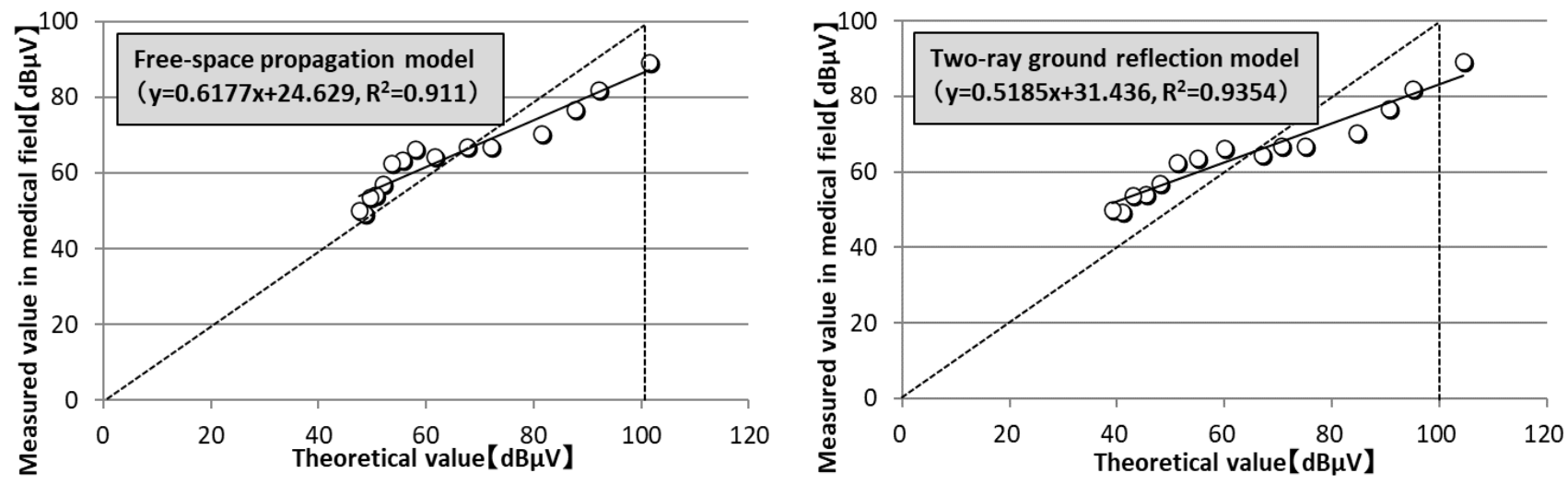

Fig. 5: Regression analysis results for the measured values in the medical field and those obtained from the radio wave propagation model.

\section{Discussion}

a) Comparison of radio wave outputs when using radio wave propagation model and measured values in the medical field

With regard to the relationship between the measured values in the medical field and those obtained from the radio wave propagation model, it was proposed from the results of the regression analysis that both models can be used for threshold estimation of the distance characteristic.

In contrast, we focus on comparison of the distance characteristics of the measured values in the medical field with those obtained from the radio wave propagation model. In both the free-space propagation model and the two-ray ground reflection model, a reversal of the measured value occurred in the medical field near the break point. From the above, it is considered that this represents the tendency changing between the short distance and long distance conditions. When compared with the radio wave propagation model values, the measured values in the medical field tended to be low at short distances and high at long distances. The break point is the point where the influence of the Earth begins to appear in the first Fresnel zone. It is considered to be important as a guide to the points that represent the change between short distance and long distance conditions.

b) Quantitative evaluation method of radio wave output of WMTT in the medical field

Based on the above, we make the following assumptions during measurement of the radio wave output of the WMTT in the medical field. Two specific situations were considered: measurement in the medical equipment management room and measurement in the ward. Because it is important to understand the break point in both situations, the break point was calculated in advance.

First, when taking the measurements in the medical equipment management room, it was assumed that the propagation distance would be smaller than the break point. This is because the medical equipment management room is often small. Therefore, given that the measured value was slightly lower than the calculated value obtained from the radio wave output using the radio wave propagation model, the threshold value was set based on the propagation distance. 
Second, when taking the measurements in a ward, it was assumed that the propagation distance will be greater than the break point. This is because the measurements are taken in a larger space. Therefore, given that the measured value was slightly higher than the calculated value obtained from the radio wave output using the radio wave propagation model, the threshold value was set based on the propagation distance.

\section{c) Limitations of this study}

This study has some limitations. First, there is a problem with the experimental environment. This experiment was performed with an antenna height of 1 $\mathrm{m}$, a corridor width of $2.8 \mathrm{~m}$ (distance in the horizontal direction to the measurement point: $1.4 \mathrm{~m}$ ) and a ceiling height of $2.6 \mathrm{~m}$ (distance in the vertical direction from the measurement point: $1.6 \mathrm{~m}$ ) only. In contrast, in the actual measurement environment, the distances to the wall surfaces, the floor surface and the ceiling are varied. It is undeniable that the measured value may show different tendencies if the environment is different.

Second, there is the problem of the antenna gain. In this work, the antennas were assumed to be isotropic antennas and the theoretical values were calculated on this basis. Although the isotropic antenna is a virtual antenna, it is very difficult to grasp the antenna gain accurately in the medical field. Therefore, we adopted a method that any researcher can calculate easily.

Finally, there is a problem with the WMTT model used. In the experiments, only one model of WMTT produced by one manufacturer was tested. The actual measurement values would undoubtedly differ when obtained using equipment from other manufacturers and different models.

\section{Conclusion}

In this work, while assuming line-of-sight propagation, we have demonstrated the possibility of quantifying radio wave output in the medical field using two types of radio wave propagation model: a freespace propagation model and a two-ray ground reflection model. To determine a reference threshold value when using each model, determination of a break point is important to grasp the change point between the short distance and long distance conditions.

It was found that the measured value was lower than the theoretical value below the break point, but the measured value was then slightly higher than the theoretical value beyond the break point.

\section{ACKNOWLEDGMENT}

We thank David MacDonald, MSc, from Edanz Group (https://en-author-services.edanzgroup.com/ac) for editing a draft of this manuscript.

\section{References Références Referencias}

1. Ministry of Health, Labor and Welfare. (2007). The fifth revision of the medical law. (in Japanese).

2. Electromagnetic Compatibility Conference Japan. (2016). Guidance for using a radio wave securely and safety in a hospital. Association of Radio Industry and Business, Tokyo. Retrieved from http://www.emcc-info.net/info/info280404.html (in Japanese).

3. E. Hanada, K.ishida, T.Kudou. (2018). Newly identified electromagnetic problems with medical telemetry systems. Przeglad Elektrotechniczny, 2, 21-24.

4. K. Ishida, et al (2018). Electromagnetic compatibility of wireless medical telemetry systems and lighting-emitting diode (LED) lamps. Przeglad Elektrotechniczny, 2, 25-28.

5. K. Fujii, Y. Ohno, M. Kido, K. Ishida, H. Jeong. (2018). Effect of Wandering Sensing Systems on Wireless Medical Telemetry Systems. Japan Journal of Medical Informatics, 38(6), 321-336.

6. S. Yamaguchi, M. Hayashi, T. Komatsu, et al. Transmission range of the wireless medical telemetry system and its dager. (2010). The Japanese journal of medical instrumentation, 80(5), 171-172. (in Japanese).

7. Association of Radio Industries and Businesses. (2008). Medical Telemeter Radio Equipment for Specified Low Power Radio Station. (in Japanese).

8. Y. Karasawa. Radiowave propagation fundamentals for digital mobile communications (second edition). Corona publishing co., Itd., 15-21. (in Japanese).

9. J. Takada. (2004). Basic theory of radio wave propagation. Microwave Workshops and Exhibition 2004 Tutorial Lecture. Retrieved from https://www.apmc-mwe.org/mwe2005/src/TL/TL0501.pdf (in Japanese). 